\title{
Inspection of silver-sinter die attaches by pulsed and lock-in infrared thermography with flash lamp and laser excitation
}

\author{
D. R. Wargulski*, D. May ${ }^{\star *}$, C. Grosse-Kockert*, E. Boschman**, B. Wunderle ${ }^{\star *}$ and M. Abo Ras* \\ * Berliner Nanotest und Design GmbH, Volmerstraße 9b, 12489 Berlin, Germany, wargulski@nanotest.eu \\ ** Chemnitz University of Technology, Chemnitz, Germany \\ ${ }^{\star * *}$ Advanced Packaging Center, Duiven, Netherlands
}

\begin{abstract}
In this study we demonstrate the capabilities of the IR thermography for the inspection of silver-sinter die attaches. Sintered specimens such as $15 \times 15 \mathrm{~mm}^{2}$ dummy dies sintered on AMB substrates have been measured by pulsed infrared thermography (PIRT), lock-in infrared thermography (LIT) and as a reference by scanning acoustic microscopy. PIRT and LIT will be compared with the focus on the applicability in production lines. Both methods usually need a sample surface blackening which will be avoided here. This need of a sample preparation by blackening or other coating for sample surfaces with low emissivity and absorptivity is one of the main reasons for the low technology adoption of thermography methods for the inspection of thermal interfaces in electronics in industry.
\end{abstract}

\section{Introduction}

Silver-sintering is a technology with increasing importance for the industry, especially in the fields of packaging and power electronics [1]. Due to the enhanced thermal performance and improved reliability the sinter technology will most probably replace the conventional soft soldering in many fields of electronics. But with new technologies new challenges appear. For saving costs and resources and to improve the reliability the industry wants to test $100 \%$ of produced electronic components and every sintered layer on it. The inspection methods for such in-line inspections are rare. For most production lines the scanning acoustic microscopy is not fast enough, and the need of a coupling medium such as water is not favorable for electronics. X-ray based methods are expensive but very common and are working very well for solder layers but for sintered layers with delamination as a common defect such methods are not the best choice since delaminations are in most cases not detectable by x-ray methods. Therefore, the IR thermography is a suitable solution. Pulsed IR thermography (PIRT) and lock-in thermography (LIT) are two non-destructive testing methods capable to detect delamination and voiding in sinter and solder layers.

\section{Methodology}

\subsection{Pulsed infrared thermography (PIRT)}

Pulsed infrared thermography is a very fast and non-destructive testing technique. It is relatively common to use it for the inspection of large production parts such as composites [2, 3], but it can be applied as well for the detection of defects in die attaches and other thermal interfaces. In this application PIRT can detect delamination and voids below dies in silver-sintered layers.

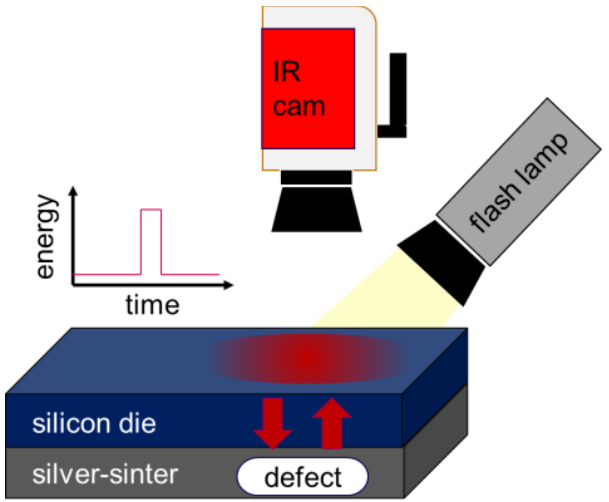

Figure 1: Principle and setup of a pulsed infrared thermography measurement on a sintered die

The Figure 1 shows the setup and principle of a PIRT measurement on a sintered die with a defect in the sinter layer. The excitation by a short pulse of light, generated by a flash lamp, leads to a heating of the sample surface. As a part of the following surface cooling process the heat diffuses through the die to the silver layer. In case of an intact sinter 
layer the heat can further diffuse to the substrate, but if a defect such as a void or delamination appears this heat is trapped. The result is a heat accumulation above the defect which can be detected by the IR camera as surface areas with increased temperature and a contrast, respectively [4].

The main advantage is the short duration of a measurement which is easily done within a second. Since the defect contrasts are usually relatively low, those signals need to be amplified. In conventional thermography measurements on such samples this will be done by spray-coating with black camera varnish or graphite. The black coating increases the absorption of the flash excitation and therefore the resulting defect signal. But the main benefit of such a coating is the emissivity increase of the sample surface, the property to emit thermal radiation. This boosts the thermal signal which is detectable by the IR camera. Especially in cases of silicon dies or metallization, by coating the signal increases by a multiple and many defects are therefore not detectable without coating [5-7].

\subsection{Lock-in infrared thermography (LIT)}

LIT is a well-known non-destructive testing method. In this case a laser serves as an excitation source to heat up the sample surface periodically. The advantages of a laser compared to e.g. halogen lamps are the high energy densities, high modulation frequency and the availability of different wavelengths. This allows the best possible laser to be selected for the respective sample surface.

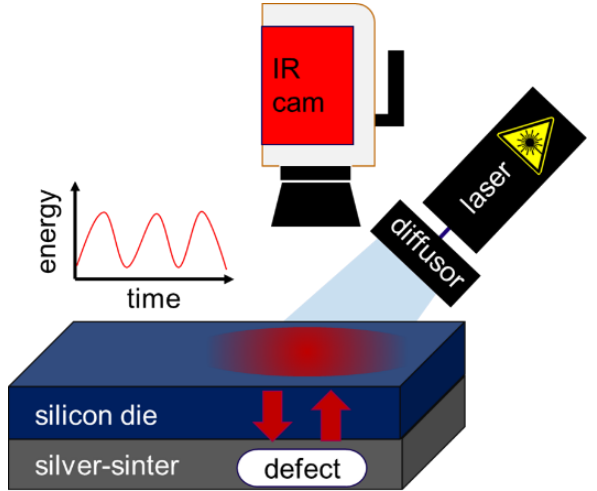

Figure 2: Principle and setup of a lock-in thermography measurement on a sintered sample

The principle of defect detection is similar to PIRT and illustrated in Figure 2. Disturbances of the cooling process in cases of defects generates a thermal pattern on the sample surface. Here again, an IR camera records this signature on the sample surface, an oscillating temperature field. The data will be lock-in amplified to increase signal to noise ration and thus detect the temperature variations caused by defects [4]. Another very important result of lock-in amplification is the phase information. As will be shown later, a disturbance in the cooling path causes a phase shift. This makes the LIT particularly suitable for weak thermal signals. However, at the price of a slightly longer measuring time.

\subsection{PIRT and LIT systems and the sintered samples}

The PIRT measurements have been performed with a complete off-the-shelf solution, which is shown in Figure 3a. This setup consists in general of a small robust industrial IR camera, an $1 \mathrm{~kJ}$ excitation flash lamp and a vacuum lamination system for measurements of low-emissivity samples, which are not allowed to be contaminated by highemissivity spray coatings such as graphite.
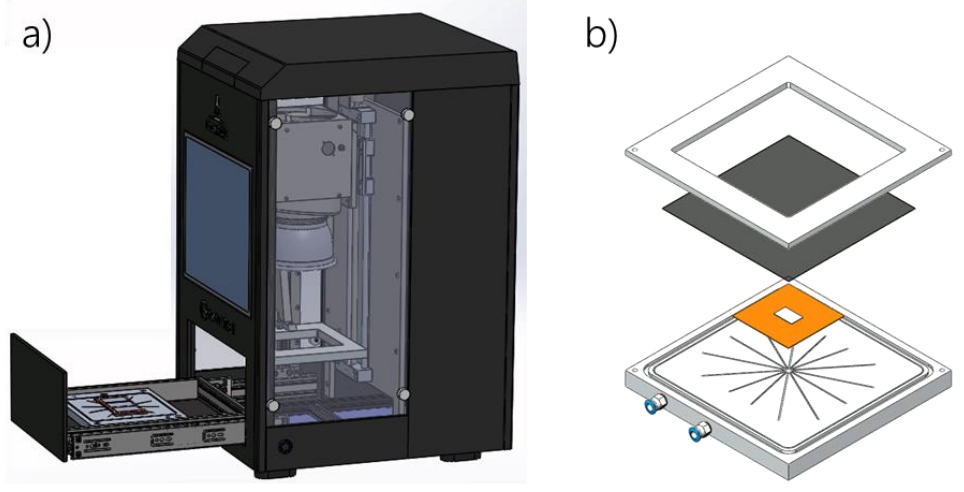

Figure 3: a) PIRT system with integrated IR camera and excitation flash lamp. b) Principle of the foil lamination technique to avoid sample blackening 
This vacuum lamination system, shown in Figure $3 \mathrm{~b}$, consists of a base with venting slits and connections to a vacuum pump. This base is simultaneously the sample table. During a measurement, a special high-emissivity foil, and a frame for the sealing drives down to the sample. The foil attaches (thermally) to the sample due to the vacuum and the measurement can start.

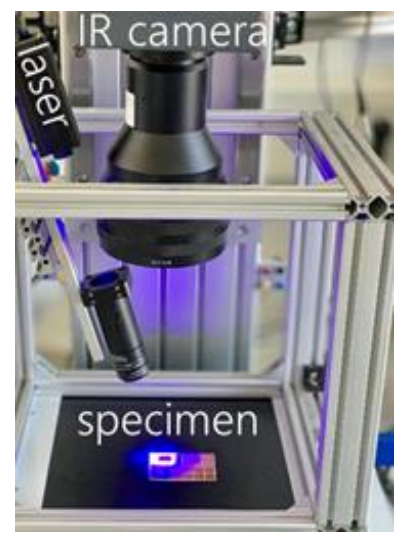

Figure 4: Laboratory setup for LIT measurements with laser excitation

The second system consists of a laboratory setup with a high-end IR camera and a $5 \mathrm{~W}$ diode laser (450 nm) as excitation source. The proprietary camera software was capable to control the laser modulation for any frequency and performing the lock-in amplification. For a comparison, this setup was combined with a transportable vacuum lamination system.

For this study three samples have been produced with different induced defects. They consist of $15 \times 15 \mathrm{~mm}^{2}$ dummy silicon dies and have been silver-sintered on active metal brazed (AMB) substrates. For the confirmation and definition of the implemented defects the sample have been analyzed by a very common inspection technique for thermal interfaces, the scanning acoustic microscopy (SAM).
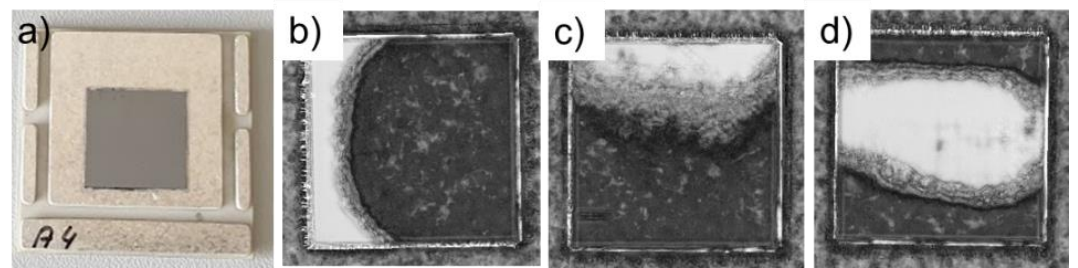

Figure 5: a) Photo of one of the three samples and b-d) the results of the SAM imaging

Figure 5 show a photo of one of the analyzed samples and the three SAM images, which clearly show delamination and voiding in the sinter layer in white and light grey.

\section{Results}

The Result of PIRT is a sequence of thermal images generated at different times after the excitation, where the image with the highest defect contrast needs to be found. Which image it is depends on the material above the defect and the depth of the defect. The images with the highest contrast from the measurements sequence are shown in Figure 6.
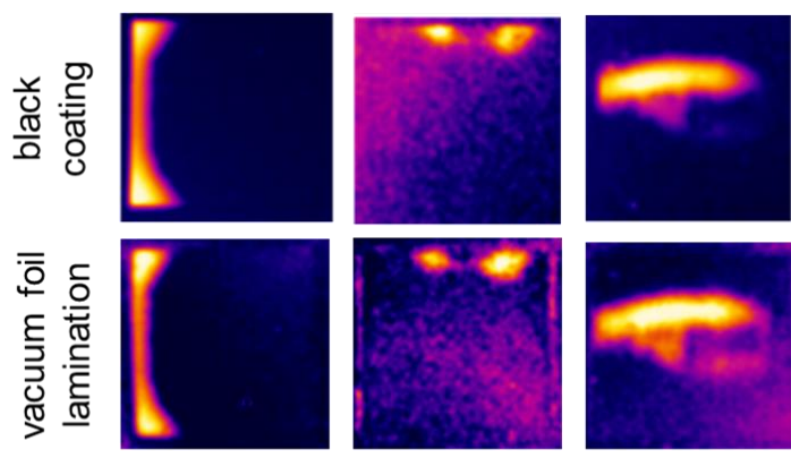

Figure 6: PIRT results of the samples coated with black camera varnish compared to uncoated samples measured with the vacuum foil lamination system 
Untreated samples can not be measured by the PIRT system since the reflective low- emissivity surface of the silicon dies. Due to the low flash energy absorption and the low signal emission at the same time, the camera is not able to detect a defect signal. The conventional procedure to face this problem is the coating with black high-emissivity spraycoatings such as camera varnish or graphite sprays. They can work very well with contrast rich IR results which are shown in Figure 6. But the spray-coating is time-consuming since the coating needs to dry and the samples after the measurements cleaned. Especially for inline inspections in the industry this specimen contamination is not acceptable. Therefore, the vacuum foil lamination technique has been developed. The vacuum ensures a suitable thermal contact between the sample surface and the thin foil to efficiently conduct the defect signal. Since the foil has a very high emissivity compared to the blank die surface, the defect signal can be strongly emitted as thermal radiation and recorded by the IR camera. Figure 6 shows the results which are competitive in quality and contrast to the conventional coated samples. Both measurements correspond very well to the SAM images in Figure 5, even if the defects appearing larger in the SAM images which could be an image scaling issue.

The results of LIT measurements are two images, an amplitude image, and a phase image, both with different advantages. The phase images, for example, are less sensitive to disturbing surface defects, reflections and other artefacts, which is the reason that the following results are just shown as phase images. Analogous to the choice of the right image in a PIRT image sequence, in LIT measurements the optimal choice of frequency is inevitable since the sensing depth is frequency dependent. The frequency needs to be low enough to reach the defects in the sinter layer and simultaneously the thermal excitation amplitude is higher due to longer heating times per period. But this comes with the cost of a longer measurement time, which is a critical parameter in industry. The described effect is illustrated in Figure 7. One sample with a typically shaped delamination at the left-hand side has been measured at 1, 2, 5 and $10 \mathrm{~Hz}$. The graph shows an exponential decay of the defect contrast with increasing frequency which is in phase images represented by the phase contrast or phase shift. In the inset phase images the decreasing signal-noise-ratio is visible with increasing frequency.

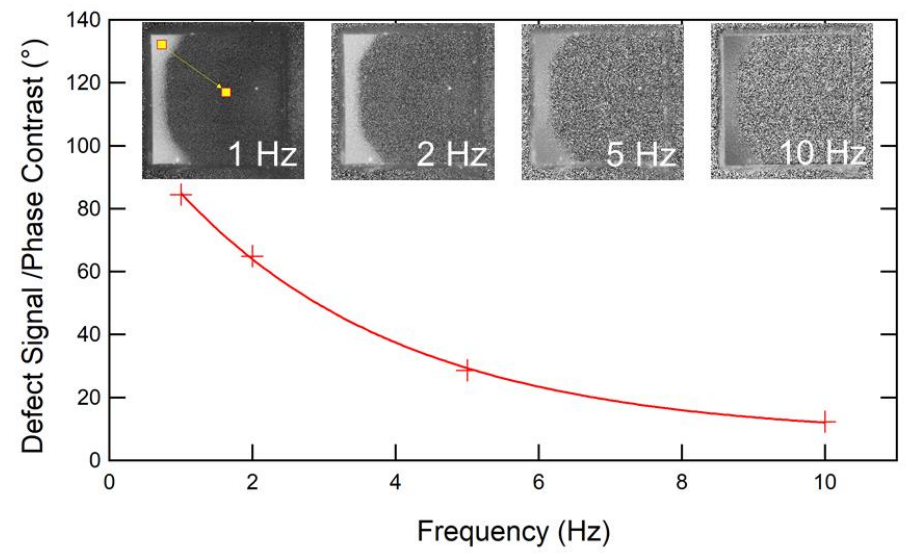

Figure 7: Phase contrast for different LIT excitation frequencies from 1 to $10 \mathrm{~Hz}$. Better SNR and phase contrast for lower frequencies. The yellow squares and arrow in the inset image are indicating the measurement points for the contrast determination.

Compared to the PIRT system the LIT system was capable to perform measurements without any sample preparations. The reason is the high-end research IR camera with a higher sensitivity and the applied lock-in amplification which enables the detection of signals which are usually below the signal-noise-ratio.
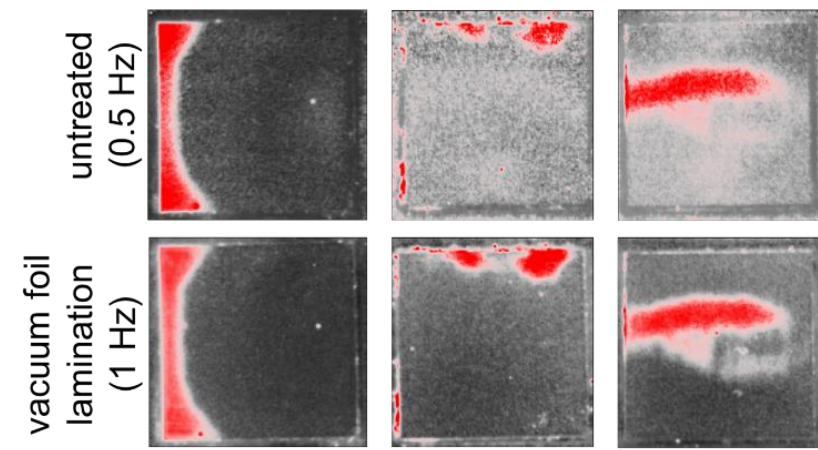

Figure 8: LIT results of the untreated samples measured at $0.5 \mathrm{~Hz}$ compared to measurements at $1 \mathrm{~Hz}$ with the foil lamination system. 
Figure 8 shows the comparison of LIT measurements with completely untreated samples and with the application of the vacuum foil lamination. It is remarkable that LIT by laser excitation is able to measure such reflective samples. The results are showing all previously measured defects but with the cost of long measurement times and a low frequency of just $0.5 \mathrm{~Hz}$. The images with vacuum lamination show images with higher contrasts and defect signals due to the higher emissivity of the foil even with a double as high frequency. The results of the laminated sample in the PIRT measurements and in the LIT measurements are comparable and the qualitative differences are not significant.

\section{Conclusions}

In conclusion, both methods, PIRT and LIT, are capable of detecting defects such as voids and delamination below dies in silver-sintered layers with an accuracy comparable to scanning acoustic microscopy. The important advantages of PIRT and LIT compared to scanning acoustic microscopy are their in-line capability. In this work we could show that PIRT and LIT can be applied for sintered specimen in industrial production lines, since the measurement duration is short enough and inline inspections are possible without sample contamination by water (SAM) or high-emissivity coatings (conventional IR thermography). Due to the newly developed vacuum lamination technique PIRT measurements can be performed without sample preparations/coatings and can increase the signal strength in LIT measurements compared to completely untreated samples.

\section{REFERENCES}

[1] M. Schaulin, M. Oppermann, T. Zerna, "Thermographic inspection method for quality assessment of power semiconductors in the manufacture of power electronics modules", 7th Electronic System-Integration Technology Conference (ESTC), 2018

[2] F. Ciampa, P. Mahmoodi, F. Pinto and M. Meo, "Recent Advances in Active Infrared Thermography for NonDestructive Testing of Aerospace Components", Sensors (2018), 18:609

[3] F. Weritz, R. Arndt, M. Röllig, C. Maierhofer, H. Wiggenhauser, "Investigation of concrete structures with pulse phase thermography", Mat. Struct. (2005) 38: 843

[4] D. May, B. Wunderle, R. Schacht, und B. Michel, „Transient thermal response as failure analytical tool - a comparison of different techniques", in Thermal, Mechanical and Multi-Physics Simulation and Experiments in Microelectronics and Microsystems (EuroSimE), 2013 14th International Conference on, Apr. 2013

[5] D.R. Wargulski, D. May, F. Löffler, T. Nowak, J. Petrick, C. Grosse, B. Wunderle, E. Boschman, and M. Abo Ras. An In-line Failure Analysis System Based on IR Thermography Ready for Production Line Integration. Proc. 25th Therminic Conf., Lecco, Italy, Sept 25-27, 2019.

[6] D.R. Wargulski, F. Löffler, D. May, J, Heilmann, B. Wunderle, A. Ziaei, and M. Abo Ras. Comparative DieAttach Failure Analysis by Thermoreflectance, Infrared Thermography and Scanning Acoustic Microscopy. Proc. 24th Therminic Conf., Stockholm, Sweden, Sept. 26-28, 2018.

[7] D. May, S. Fritzsche, A. Cardoso, R. Schacht, und B. Wunderle, „IR pulse thermography as failure analytical tool applied to die attach processes“, in 2015 21st International Workshop on Thermal Investigations of ICs and Systems (THERMINIC), Sep. 2015. 